\title{
Retrospective Analysis of Adherence to Thromboprophylaxis after Orthopedic Surgery in a Community Hospital
}

\author{
Megan A McElwee, Aaron Tejani, and Lily Cheng
}

$\mathrm{V}$ enous thromboembolism is a well-known complication of total knee replacement, total hip replacement, and hip fracture repair. Venous thromboembolism, which includes deep vein thrombosis and pulmonary embolism, can cause significant morbidity and mortality. ${ }^{1}$ In addition, treatment of this condition can result in substantial costs to the health care system. ${ }^{1,2}$ Patients who have undergone orthopedic surgery are considered at high risk for these complications, with the rate of venous thromboembolism approaching $40 \%$ to $60 \%$ among patients who have not received postoperative thromboprophylaxis. ${ }^{1}$ Rates of venous thromboembolism have decreased significantly with the use of guideline-recommended thromboprophylaxis. ${ }^{1}$ Because many cases of venous thromboembolism occur after discharge from hospital, postdischarge prophylaxis is an important component of treatment. ${ }^{1}$

Thromboprophylaxis for patients who have undergone orthopedic surgery has been the standard of care for more than 15 years. ${ }^{1,3}$ The 2008 guidelines of the American College of Chest Physicians (ACCP) recommended the use of a low molecular-weight heparin (LMWH), fondaparinux, or a vitamin $\mathrm{K}$ antagonist (e.g., warfarin) for at least 10 days after total knee replacement and for an extended period of up to 28-35 days after total hip replacement or hip fracture repair. ${ }^{1}$ Although patients who have undergone orthopedic surgery are generally considered to be at high risk for venous thromboembolism, individual patient risk factors also influence the occurrence of this problem. ${ }^{1}$ Additional risk factors include immobility, cancer, previous venous thromboembolism, older age, and obesity. ${ }^{1}$ Risk stratification has historically been used to make decisions about thromboprophylaxis for individual patients; however, such stratification is often not done in practice because it is quite cumbersome. ${ }^{1}$

With the availability of LMWH, the use of warfarin has been decreasing steadily in this patient population, which avoids the need to monitor the international normalized ratio (INR) and titrate warfarin to a target INR of $2-3 .{ }^{1}$ However, several barriers still exist to providing appropriate thromboprophylaxis with LMWH in this patient population. Because thromboprophylaxis of at least 10 days (for total knee replacement) and of extended duration (for total hip replacement or hip fracture surgery) has been shown to reduce the risk of venous thromboembolism, it is remarkable that more patients are not receiving postdischarge therapy, ${ }^{1,3}$ especially given that a recent meta-analysis showed no significant increase in episodes of major bleeding when these agents were used for this purpose. ${ }^{3}$

The results of studies evaluating in-hospital adherence to available guidelines have been disappointing, with most adherence rates being suboptimal. ${ }^{4-7}$ Adherence with postdischarge thromboprophylaxis is also important, given reports that the majority of cases of thromboembolism after total hip and knee replacement are diagnosed after discharge. ${ }^{8}$

The primary objective of this retrospective analysis was to examine the rate of adherence to the 2008 ACCP guidelines for the appropriate duration of postoperative therapy with LMWH or warfarin among orthopedic patients in an urban community hospital. Previous studies of adherence have rarely reported postdischarge compliance, but for this study, in-hospital data were combined with community data to generate the total duration of therapy.

\section{METHODS}

This study was conducted at Burnaby Hospital, a 267-bed urban hospital in southwestern British Columbia. The 2008 ACCP guidelines ${ }^{1}$ were used in formulating the data collection form, which stratified patients according to the type of surgery they had undergone and their individual risk factors. The patients were stratified into 4 groups (designated 0, 1, 2, and 3) according to their additional risk factors for venous throm- 
boembolism (see Appendix 1). These categories were used to determine if patients at higher risk for venous thromboembolism received an extended duration of thromboprophylaxis (> 10 days). An observational analysis was conducted to determine the duration of therapy (up to 10 days, $10-27$ days, or more than 27 days) for patients undergoing each type of surgery (total hip or knee replacement or hip fracture surgery). The rationale for this analysis stemmed from the ACCP guidelines, ${ }^{1}$ which recommended that all patients receive thromboprophylaxis for at least 10 days, along with a strong suggestion that thromboprophylaxis be continued for up to 35 days. Clinical trials have studied extended-duration prophylaxis ranging from 28 to 35 days. $^{3}$ For the current study, the intention was to determine if the duration of thromboprophylaxis was variable and if any such variation could be related to the type of surgery.

The project received approval from the Research Ethics Board of the Fraser Health Authority before initiation of data collection.

To be included in the study, patients had to be older than 18 years of age and had to have undergone total hip or knee replacement or hip fracture surgery at the Burnaby Hospital during the defined study period of March 30, 2007, to April 1, 2008. The Decisions Support Department provided a list of all patients meeting these inclusion criteria. Based on published adherence rates of $50 \%$, a sample size of 200 patients was necessary to have $95 \%$ confidence in choosing a sample of patient charts that would adequately represent all patients who underwent total hip or knee replacement or hip fracture surgery during the study period.

Patients were excluded if they had been taking anticoagulants before admission or if they had a bleeding disorder that would contraindicate the use of anticoagulants (hemorrhagic diathesis, severe thrombocytopenia, suspected intracranial hemorrhage, active ulcerative lesions, or hemophilia).

The sample cohort of patients was selected using a stratified systematic sampling method. A total of 114 total hip replacements, 258 total knee replacements, and 138 hip fracture surgeries had been performed at the hospital during the study period. To ensure a cohort of patients that would be representative of the entire sample, it was determined that the sample should consist of about 67 patients who had undergone total hip replacement, 73 patients who had undergone total knee replacement, and 70 patients who had undergone hip fracture surgery, assuming at least 50\% adherence to the guidelines. Subsequent to the stratified systematic sampling, the final cohort consisted of 194 patients. To select patients who had undergone total hip replacement or hip fracture surgery, every second patient was selected from the respective lists of patients who had undergone these procedures during the study period. To select patients who had undergone total knee replacement, every fourth patient was selected from the list of patients had undergone total knee replacement during the study period. The principal investigator (M.A.M.) reviewed the charts of the selected patients to determine the duration of thromboprophylaxis after surgery but before discharge from the hospital. Information about the agent used in the hospital was collected, and risk factors were assessed (see Appendix 1).

Information about thromboprophylaxis after discharge from the hospital was obtained from PharmaNet, a provincewide network that holds a central database of all prescription medications dispensed in British Columbia. ${ }^{9}$ These data reflected the duration of use of thomboprophylactic medication dispensed from community pharmacies after hospital discharge. Following the chart review, a cohort file including specified patient information (personal health number, number of days of thromboprophylaxis in the hospital, index date [date of discharge], code for surgery type, code for risk level, and thromboprophylactic agent used in the hospital) was sent to PharmaNet. PharmaNet staff then calculated each study patient's total duration of thromboprophylaxis, by summing the days of therapy in the hospital and the days of therapy in the community (i.e., days' supply of anticoagulants represented by prescriptions filled after discharge). To preserve patients' confidentiality, the cohort file was then stripped of the patients' personal health number and the duration of thromboprophylaxis received in the hospital, and the calculated data were returned to the investigators. Information received back from PharmaNet consisted of a study identification number (to replace each patient's personal health number), total duration of thromboprophylaxis therapy (days), surgery code, risk code, index date (date of discharge), anticoagulant used, quantity of medication, and days supply. With the information provided by PharmaNet, it was possible to determine the rate of adherence to the 2008 ACCP guidelines for duration of thromboprophylaxis. It was also possible to determine the trends in prescribing for patients with various levels of risk associated with these procedures. Descriptive statistics were used to summarize the results.

\section{RESULTS}

A total of 194 selected charts met the inclusion criteria and were reviewed for potential exclusion criteria. Twenty-four of the patients were excluded. Of the patients who had undergone total knee replacement, 7 were excluded because they were receiving anticoagulation before admission, and 1 was excluded because of an order for 6 months of anticoagulation therapy in the community to treat deep vein thrombosis that occurred in the hospital. Of the patients who had undergone total hip replacement, 7 were excluded because they lacked a personal health number (out-of-province patients), 1 was excluded because of active gastrointestinal bleeding, and 1 was excluded 
because of anticoagulation before admission. Of the patients who had undergone hip fracture surgery, 4 were excluded because of active gastrointestinal bleeding, 2 were excluded because of anticoagulation before admission, and 1 was excluded secondary to thrombocytopenia. This left a cohort of 170 patients: 56 who had undergone total knee replacement, 51 who had undergone total hip replacement, and 63 who had undergone hip fracture surgery. Although this cohort was not large enough to meet the predetermined sample size, it was sufficient to generate $95 \%$ confidence that the 170 selected charts were a representative sample of the 510 patients who underwent total hip or knee replacement surgery or hip fracture repair during the study period (with a margin of error of $\pm 6.02 \%$, with the identified guideline adherence rate of approximately $40 \%$; see below).

Only $28(44 \%)$ of the 63 patients who underwent hip fracture surgery, $24(47 \%)$ of the 51 patients who underwent total hip replacement, and $21(38 \%)$ of the 56 patients who underwent total knee replacement received the minimum recommendation of at least 10 days of thromboprophylaxis (Table 1). A separate analysis of in-hospital data revealed that only 25 (40\%) of the 63 patients who underwent hip fracture surgery, $6(12 \%)$ of the 51 patients who underwent total hip replacement, and $2(4 \%)$ of the 56 patients who underwent total knee replacement received prophylaxis for at least 10 days during their hospital stay; most patients were discharged before they had received the minimally recommended duration of therapy.

An observational analysis by risk category showed no apparent correlation between extended duration of thromboprophylaxis and patients' associated risk factors for venous thromboembolism (Table 2). Despite their being in the highest-risk group, $62 \%$ of the patients at risk level 3 who underwent hip fracture surgery, $50 \%$ of the patients at risk level 3 who underwent total hip replacement, and $62 \%$ of the patients at risk level 3 who underwent total knee replacement received less than the recommended minimum of 10 days of thromboprophylaxis. In fact, only 23\%, 14\%, and $8 \%$ of the patients with risk level 3 in the groups who underwent hip fracture surgery, total hip replacement, and total knee replacement, respectively, received the recommended extended duration of thromboprophylaxis (greater than 27 days).

\section{DISCUSSION}

This retrospective analysis of thromboprophylaxis fills several gaps in the literature. It is the first study of its kind in British Columbia, and the study population encompassed all adults, not just elderly patients. Hospital pharmacists are usually aware of the prescribing patterns in their respective institutions, but often little is known about what occurs once patients are discharged. This study offered the benefit of observing what was happening outside of the hospital, after patients were discharged. Use of the PharmaNet database allowed us to determine the total duration of thromboprophylactic therapy, which in turn enabled us to observe gaps in adherence to the recommended guidelines. These results showed poor adherence to the recommended guidelines and revealed that patients were receiving suboptimal therapy. Although most patients received at least some thromboprophylaxis, most did not receive an adequate duration of therapy. We strongly suspect that this problem exists in other jurisdictions in Canada, and we therefore emphasize the need for similar audits at other hospitals that provide orthopedic surgery services.

These results are consistent with those for similar audits conducted with comparable objectives in mind. A survey of 397 Canadian orthopedic surgeons reported that only $36 \%$ of physicians ordered postdischarge thromboprophylaxis for their patients. ${ }^{4}$ A 2008 retrospective clinical audit of adherence to a thromboprophylaxis protocol for surgical patients found that only $29 \%$ of patients received adequate therapy. ${ }^{1}$ An audit of various hospitals in the United States examined compliance with the ACCP guidelines and found that of 2324 patients admitted for orthopedic surgery, $36.8 \%$ had received an inadequate duration of thromboprophylaxis and 56.3\% had received no prophylaxis. ${ }^{5}$ Another recently published study based on a large US health claims database (and reported in abstract form $)^{6}$ found that only $40 \%$ of 3497 patients who underwent orthopedic surgery received the appropriate thromboprophylaxis as described by the ACCP guidelines.

Through this study, we sought to provide other sites offering orthopedic surgery with a framework to assess the level of adherence to thromboprophylaxis for their patients. Our suggestion is that all sites complete similar audits using inhospital and postdischarge data. In addition, future studies should include a component to identify the reasons for nonad-

Table 1. Duration of Thromboprophylaxis

Type of Surgery; No. (\%) of Patients

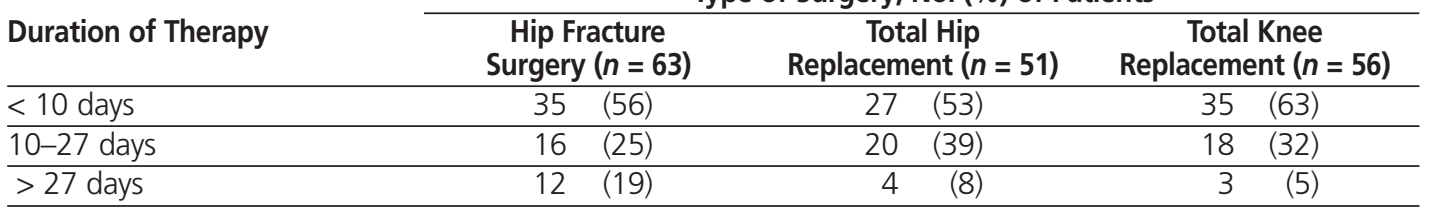


Table 2. Duration of Thromboprophylaxis with Risk Stratification

\begin{tabular}{|c|c|c|c|}
\hline \multirow[b]{2}{*}{ Risk Level* } & \multicolumn{3}{|c|}{ Duration of Therapy; \% of Patients } \\
\hline & $<10$ Days & 10-27 Days & > 27 Days \\
\hline \multicolumn{4}{|c|}{ Hip fracture surgery } \\
\hline 0 & 100 & 0 & 0 \\
\hline 1 & 44 & 36 & 21 \\
\hline 2 & 67 & 0 & 33 \\
\hline 3 & 62 & 15 & 23 \\
\hline \multicolumn{4}{|c|}{ Total hip replacement } \\
\hline 0 & 71 & 29 & 0 \\
\hline 1 & 53 & 37 & 11 \\
\hline 2 & 45 & 55 & 0 \\
\hline 3 & 50 & 36 & 14 \\
\hline \multicolumn{4}{|c|}{ Total knee replacement } \\
\hline 0 & 43 & 57 & 0 \\
\hline 1 & 61 & 32 & 7 \\
\hline 2 & 88 & 13 & 0 \\
\hline 3 & 62 & 31 & 8 \\
\hline
\end{tabular}

herence, so that specific deficiencies can be addressed. For example, other sites could use a prospective observational design in which orthopedic surgery patients are followed to determine if they actually receive their prescriptions and if so, whether they go on to fill those prescriptions. In addition, patients who receive but do not fill prescriptions could be asked why they have not done so.

Many potential barriers might explain the lack of adherence evident at the authors' community hospital. These potential barriers include lack of appropriate risk stratification, lack of prescribing, lack of filling of prescriptions by patients, prohibitive cost of thromboprophylactic agents, and lack of follow-up in the community. One drawback of this study was our inability to determine whether the lack of filling of postdischarge prescriptions in the community was a result of physicians not prescribing the medications or patients not filling prescriptions. As mentioned, one barrier that might contribute to a patient's decision to not fill a prescription could be the cost of LMWH. Despite guidelines recommending up to 28-35 days of therapy for high-risk patients, the provincial drug coverage program provides funding for only up to 10 days of therapy. ${ }^{10}$ The increased workload and transition to INR monitoring in the community for patients receiving vitamin $\mathrm{K}$ antagonists may also be a barrier. Other potential barriers could be physicians' heightened level of concern regarding their patients' risk of bleeding with these agents, lack of awareness of current guidelines, and individual physicians' prescribing patterns. Further research may be warranted to determine the specific barriers that are causing this lack of adherence, to allow implementation of effective changes to current practice in the authors' institution.

Recommendations that could be considered to improve adherence in the future include creation of preprinted order sets incorporating the hospital's policy for use of these agents, preprinted discharge prescriptions, further involvement of pharmacy staff, education of surgeons and patients, better criteria for determining the risk of bleeding (in relation to potential benefit of thromboprophylaxis), and individual site audits (including follow-up audits). ${ }^{6}$ In addition, more research is needed to identify specific areas where change is needed, as each hospital may have particular reasons for nonadherence.

This study had some limitations. For example, some patients may have been discharged from one hospital to another hospital, rather than to home, but because PharmaNet is a community-based system, we would have been unable to track prescriptions filled at another facility. With oral tablets of multiple strengths (e.g., warfarin), it is sometimes difficult to determine the duration of therapy, as patients may be taking more than one tablet per dose. Therefore, even though we were able to determine the filling of these prescriptions, this could represent a source of error. The intent was to analyze information for 200 patients, but only 170 patients were included. However, this did not compromise the reliability of the data. In consultation with a statistician, we had $95 \%$ confidence $( \pm 6.02 \%$, instead of the prespecified $\pm 5 \%)$ that the sample of 170 patients was representative of the 510 patients who underwent total hip replacement, total knee replacement, or hip fracture repair during the study period. Finally, these findings apply only to the situation at Burnaby Hospital and may not necessarily reflect practice in other jurisdictions.

In conclusion, adherence to the 2008 ACCP guidelines for duration of thromboprophylaxis after orthopedic surgery was suboptimal at the authors' community hospital. The methodology used in this study could be used by other hospitals to assess compliance with recommendations during the hospital stay and after discharge. Future studies examining this issue 
should consider methods that allow identification of barriers to adherence, including barriers to the prescribing and procurement of therapy for use after discharge.

\section{References}

1. Geerts WH, Bergqvist D, Pineo GF, Heit JA, Samama CM, Lassen MR, et al. Prevention of venous thromboembolism: American College of Chest Physicians evidence-based clinical practice guidelines (8th edition). Chest 2008;133(6 Suppl):381S-453S.

2. Shorr AF, Nutescu EA, Farrelly E, Horblyuk RD, Happe LE, Franklin MD. Postdischarge oral versus injectable anticoagulation following major orthopedic surgery. Ann Pharmacother 2008;42(9):1222-1228.

3. Eikelboom JW, Quinlan DJ, Douketis JD. Extended-duration prophylaxis against venous thromboembolism after total hip or knee replacement: a meta-analysis of the randomised trials. Lancet 2001;358(9275):9-15.

4. Gross M, Anderson DR, Nagpal S, O’Brien B. Venous thromboembolism prophylaxis after total hip or knee arthroplasty: a survey of Canadian orthopedic surgeons. Can J Surg 1999;42(6):457-461.

5. Yu HT, Dylan ML, Lin J, Dubois RW. Hospitals' compliance with prophylaxis guidelines for venous thromboembolism. Am J Health Syst Pharm 2007;64(1):69-76.

6. Selby R, Borah B, McDonald H, Henk J, Crowther M, Wells P. Alignment to prophylaxis guidelines and occurrence of venous thromboembolism after total hip and total knee replacement [abstract]. In: Symposium of Canadian Agency for Drugs and Technologies in Health; 2009 Apr; Ottawa (ON).

7. Rahme E, Dasgupta K, Burman M, Yin H, Bernatsky S, Berry G et al. Postdischarge thromboprophylaxis and mortality risk after hip- or knee-replacement surgery. CMAJ 2008;178(12):1545-1554.

8. White RH, Romano PS, Zhou H, Rodrigo J, Bargar W. Incidence and time course of thromboembolic outcomes following total hip or knee arthroplasty. Arch Intern Med 1998;158(14):1525-1531.
9. Pharmaceutical Services Division of British Columbia. What is PharmaNet? Victoria (BC): Pharmacare Ministry of Health [revised 2008 Oct 23; cited 2009 Mar 31]. Available from: www.health.gov.bc.ca/pharme/ PharmaNet/netindex.html

10. Brand and generic prescription drug names [Special Authority criteria]. Vancouver (BC): BC Ministry of Health; [revised 2008 Oct 23; cited 2009 Mar 31]. Available from: www.health.gov.bc.ca/pharmacare/sa/criteria/ genericbrandtable.html

Megan A McElwee, BSc(Pharm), ACPR, is a Clinical Pharmacist for the Pharmacy Department, Langley Hospital, Fraser Health Authority, Langley, British Columbia.

Aaron Tejani, BSC(Pharm), PharmD, ACPR, is the Drug Information and Clinical Research Coordinator for Pharmacy Services, Fraser Health Authority, Burnaby, British Columbia.

Lily Cheng, BSC(Pharm), PharmD, ACPR, is the manager of Pharmacy Services, Burnaby Hospital and Queens Park Care Centre, Fraser Health Authority, Burnaby, British Columbia.

\section{Address correspondence to:}

Megan A McElwee

Langley Memorial Hospital

22051 Fraser Highway

Langley BC V3A $4 \mathrm{H} 4$

e-mail: Megan.McElwee@fraserhealth.ca

\section{Acknowledgements}

We thank Mary Mah (graphics and formatting), Michael Wasdell (statistical analysis), Helen Watt (PharmaNet), and Rosa Haywood (ethics), as well as staff in Fraser Health Decision Support and Burnaby Hospital Medical (data collection) Records for their help with this study.

This project was partially supported by research funds from the Pharmacy Department, Fraser Health Authority.

Appendix 1. Definitions of codes for risk levels (based on Geerts and others')

Risk factors:

- History of pulmonary embolism or deep vein thrombosis

- Active cancer or history of cancer

- Elderly (> 65 years of age):

$>75$ years

$>85$ years

$>95$ years

- Obesity (body mass index $>30 \mathrm{~kg} / \mathrm{m}^{2}$ )

- Immobility

Risk levels:

- $0=$ no additional risk factors

- 1 = mild risk ( 1 risk factor of age $>65$ years, obesity, or immobility)

- 2 = moderate risk (at least 1 of either prior pulmonary embolism, prior deep vein thrombosis, or current or prior diagnosis of cancer OR 2 risk factors of age > 65 years, obesity, or immobility)

- 3 = high risk (2 or more risk factors, including at least 1 of either prior pulmonary embolism, prior deep vein thrombosis, or current or prior diagnosis of cancer OR more than 2 risk factors of age $>65$ years, obesity, or immobility) 